\section{Fazendo política no Twitter: como os efeitos estimados das mensagens influenciam as ações e os usos da plataforma}

[Politics on Twitter: how the estimated effects of the messages influences the actions and uses of the platform]

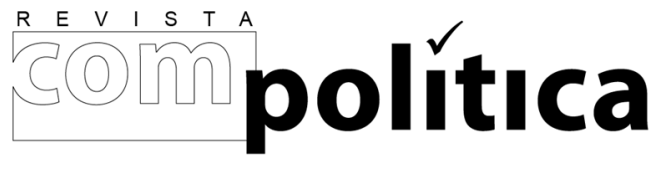

revista compolítica 2018, vol. 8(1)

compolitica.org/revista

ISSN: 2236-4781

DOI: 10.21878/compolitica.2018.8.1.141

O. Open Access Journal

\author{
Graça Penha Nascimento Rossetto \\ Centro Universitário da Bahia \\ [University Centre of Bahia]
}

\begin{abstract}
Resumo
Há algum tempo o Twitter atrai a atenção de pesquisadores da comunicação política, especialmente em relação as motivações de usos e gratificações obtidas. Entretanto, pouca atenção foi dada ao exame do efeito percebido nos usuários. Este artigo aplica a hipótese do efeito de terceira pessoa ao contexto do Twitter. A hipótese principal é que os entrevistados vão subestimar o efeito das mensagens políticas em si e superestimá-lo nos outros. $A$ plataforma foi eleita devido à utilização nas eleições e por suas características únicas. $A$ aferição empírica foi feita por meio de uma survey com usuários da plataforma, recrutados com solicitação direta e técnica snowball, entrevistados no período pós-eleitoral de 2012, resultando em 142 respostas. A hipótese principal foi confirmada e ofereceu novas descobertas para o estudo da comunicação política naquele ambiente..
\end{abstract}

Palavras-chave: efeito de terceira pessoa, comunicação política, twitter.

\begin{abstract}
For some time, Twitter attracts the attention of political communication researchers, especially on the motivations of uses and gratifications obtained. However, little attention has been given to examining the perceived effect on users. This article applies the hypothesis of the third person effect to the Twitter context. The main hypothesis is that respondents will underestimate the effect of political messages on themselves and overestimate them in others. The platform was elected due to its use in the last elections and its unique characteristics. The empirical assessment was made through a survey with Twitter's users, recruited with direct request and snowball technique, interviewed in the post-election period of 2012, resulting in 142 responses. The main hypothesis was confirmed and provided new discoveries for the study of political communication in that environment.
\end{abstract}

Keywords: third person effect, political communication, twitter. 


\title{
Fazendo política no Twitter: como os efeitos estimados das mensagens influenciam as ações e os usos da plataforma
}

\author{
Graça Penha Nascimento ROSSETTO
}

$\int \begin{aligned} & \text { á pouca dúvida quanto à internet ter afetado de diversas formas o campo da } \\ & \text { comunicação política, seja do ponto de vista da rearrumação dos atores } \\ & \text { políticos, seja pelo panorama das campanhas eleitorais ou até mesmo pela }\end{aligned}$ criação de novas formas de interação entre políticos e cidadãos.

A preocupação gira em torno de aproveitar as oportunidades de fazer-se visível, sobretudo de forma favorável, nos meios de comunicação. Esses meios incluem a internet e o que ela oferece como instrumentos para comunicação; que é onde se encaixam, entre outros, os sites de redes sociais (SRS). Esses sites são capazes de suprimir a mediação da comunicação entre o candidato e o eleitor, o que abre um novo leque de possibilidades para a prática política.

Dentre os trabalhos que tratam da interface ente política e SRS se destacam os estudos que envolvem discussão política em períodos eleitorais (WILLIAMS; GULATI, 2008; PARVIAINEN et al., 2012), ou aqueles que se debruçam sobre análises pontuais de eventos políticos de relevância (TUFEKCI; WILSON, 2012; LIM, 2012).

No âmbito das teorias da comunicação, há uma longa tradição de estudo do uso e dos efeitos dos meios de comunicação na política, especialmente sobre a televisão (CHADWICK, 2009). Com o avanço da internet, porém, essa realidade começa a mudar. Primeiro porque a rede mundial de computadores tem a capacidade de oferecer características do meio televisivo associadas à comodidade que só a internet permite. Segundo, porque ela oferece ao usuário uma possibilidade ampliada de gerar conteúdo, o que os permite subverter os protocolos de gatekeeping.

Ao nos dedicarmos aos efeitos das mensagens políticas trocadas por meio dos SRS, a literatura a qual recorremos tem suas origens nos Estados Unidos, nos anos 1930, com a Mass Communication Research. O que se extrai do evoluir histórico dessas abordagens é 
que passou-se de uma noção dos efeitos imediatos e nas ações dos indivíduos para o entendimento de que ocorrem consequências de longo prazo, influenciando, sobretudo, a construção cognitiva. Os efeitos propriamente ditos são, na verdade, uma combinação de experiências mediadas e de suas relações interpessoais (McQUAIL, 2010).

Em consonância com essa tendência está a hipótese do efeito de terceira pessoa (ETP). A abordagem sustenta que o indivíduo exposto às mensagens mediáticas acredita que o conteúdo que ele julga errado ou perigoso terá efeito nos outros, mas não neles mesmos (ou terá um efeito mais forte nos outros do que neles). Isso significa que o efeito que se verifica com esta hipótese é a influência que um efeito imaginado em terceiros exerce em nós, em nossas convicções, atitudes e comportamentos.

\section{O efeito de terceira pessoa (ETP)}

Diferente da maioria das teorias sobre os efeitos da comunicação, a hipótese do ETP não está interessada nos efeitos da comunicação sobre indivíduos (e/ou suas opiniões), mas sim no que as pessoas pensam sobre os efeitos da comunicação (PERLOFF, 2002). São essas convicções sobre a comunicação e os meios de comunicação que podem influenciar as atitudes de um indivíduo a respeito de certos tipos de conteúdo.

De forma geral, a hipótese do ETP afirma que os indivíduos são psicologicamente predispostos a superestimar os efeitos que a comunicação tem nos outros, em terceiros; e subestimar o efeito de uma mensagem mediática em si mesmo. Em última instância, tratase de um juízo a respeito da opinião alheia, uma percepção sobre o que os outros (terceiros) pensam.

A abordagem do ETP trata, assim, da opinião (pública) presumida. Esse é o ponto de maior interesse para este trabalho, já que, para a comunicação política, muitas vezes as impressões e opiniões que se tem da realidade são de fato mais importantes do que a realidade em si. Gunther et al. (2008) afirmam que a opinião percebida é importante porque é com base nela que muitas atitudes são tomadas. Crigler (2001) é ainda mais específica, relacionando o fenômeno do ETP à política: "a hipótese do efeito de terceira pessoa presume que muito 
do nosso comportamento social e político é orientado por impressões da realidade, suposições sobre o ambiente comunicacional e construções a respeito de acontecimentos sociais e políticos" (p. 179).

O primeiro estudo que apresentou a nomenclatura foi publicado em 1983, em um artigo a respeito da teoria da opinião pública do sociólogo W. Phillips Davison. De 1983 até hoje, a pesquisa sobre a hipótese do ETP contabiliza centenas de publicações sobre temas variados, desde surveys e experimentos a respeito deste efeito imaginado (ANDSAGER; WHITE, 2007; PERLOFF, 1993), passando por estudos sobre censura, violência e pornografia (SALWEN; DUPAGNE, 1999; SCHARRER, 2002), saúde (ANDSAGER et al., 2006), opinião pública e eleições (MUTZ, 1989; PRICE; STROUD, 2005) até a discussão a sobre questões metodológicas (GOLAN, 2008; PAUL et al., 2000).

O relato cronológico do que se tem pesquisado sobre o ETP indica um amadurecimento da compreensão do fenômeno e um avanço metodológico. Nos anos 80 e 90 a dedicação dos pesquisadores deu subsídio empírico para novos olhares a respeito do ETP. A partir do ano 2000, o foco deslocou-se para a avaliação das variáveis que o influenciam e a constante recomendação de observar o comportamento resultado do efeito imaginado.

É que no exame do ETP distinguem-se dois componentes. O primeiro, chamado cognitivo (perceptual component), tem a ver com a percepção que o indivíduo tem a respeito do efeito de uma mensagem nele e nos outros, é a impressão - ou percepção - do efeito sobre terceiros (third-person perception). O segundo, chamado comportamental (behavioral component), é consequência - mas consequência não essencial - do primeiro. Significa que, uma vez que o indivíduo avalia que os efeitos da mensagem são mais intensos sobre os outros do que sobre ele, toma uma atitude, muitas vezes preventiva, para evitar a realização do efeito esperado.

De acordo com Diana Mutz (1998), a percepção de terceira pessoa (third-person perception) é de uma família de conceitos científicos sociais que se concentra nas impressões de outras pessoas ou coletivos de fora do espaço da vida pessoal de um indivíduo. Essa sensação é única por centrar-se na bifurcação entre as impressões dos efeitos de mensagens sobre os outros e sobre si (a primeira pessoa). Outra característica que a torna única é o fato do conceito ser operacionalizado de forma relacional, em 
comparação. "O foco não é o efeito dos media percebido nos outros ou o efeito percebido no sujeito, mas a discrepância entre essas crenças" ${ }^{1}$ (GUNTHER et al., 2008, p. 186 tradução própria).

Os exemplos iniciais de Davison (1983) demonstram que esses "outros" podem ser mais ou menos identificáveis. Cohen, Mutz, Price e Gunther (1988) descreveram os diferentes níveis de "outros" para introduzir na literatura do ETP a noção de distância social. Ela pode ser entendida como o grau de similaridade entre mim e o outro (EVELAND et al., 1999), que pode ser referente a gostos pessoais, experiências de vida, localização geográfica, classe social, ideologia política, etc. A distância social se dá sempre em comparação e deve ser considerada em cada estudo específico, já que indivíduos podem ser socialmente próximos em relação, por exemplo, à ideologia política, mas socialmente distantes quando se trata de localização geográfica. O importante é qual característica é analisada por cada estudo. Regra geral, quanto maior a distância social identificada na pesquisa, maior a percepção do efeito de terceira pessoa.

A explicação de como exatamente o fenômeno ocorre ainda está para ser desvendada, mas a maior parte da bibliografia sugere que a resposta esteja na Psicologia; dela destacam-se duas possíveis explicações: 1) A razão de autoafirmação, ou self-enhancement motivation, que sugere que quando é benéfico não ser influenciado por determinado tipo de mensagem os indivíduos vão reportar que, de fato, não são influenciados. Ao contrário, percebem o efeito nos outros, sobretudo nos mais distantes socialmente. Essa razão fundamenta-se no desejo individual de autoafirmação e na manutenção de uma concepção positiva de si mesmo (PERLOFF, 2002); e 2) O ego-deffensive mechanism, segundo o qual quando se trata da exposição a conteúdo entendido como negativo ou inapropriado, o indivíduo tende a, através de um mecanismo de defesa do ego, não se deixar influenciar ou não sentir-se influenciado (ANDSAGER; WHITE, 2007).

De forma geral, a bibliografia sobre o ETP permite dizer que os efeitos observados são fortes (ou a discrepância entre mim e o outro é grande), sedimentados no tempo, por ele

\footnotetext{
1 "The focus is not perceived media effects on others or perceived effects on the self, but the discrepancy between these beliefs".
} 
acumulados e capazes de afetar inicialmente e, sobretudo, as convicções individuais sobre o mundo.

\section{O Twitter, a política e as eleições}

As características mais interativas da web 2.0 aparecem como rica fonte de recursos que podem ser postos a serviço da comunicação política (PARLAMEE; BICHARD, 2012; AGGIO, 2010), oferecendo potencialmente aprendizado político e formação de comportamento e atitudes. Uma replicação de mensagens no Twitter, por exemplo, revela um alargamento nas relações de contato, partilha ou interação entre os usuários.

Este diagnóstico parte do pressuposto de que os cidadãos passam a ter acesso, de forma geral, a mais informação. Na visão de Bennet e Iyengar (2008), este é o principal impacto da internet sob o campo da comunicação política: para os cidadãos, que têm oportunidade de preencher melhor seu repertório informativo; para grupos de interesse, que ampliam seus raios de ação a partir da oportunidade de interação em rede; e para os agentes políticos, capazes de organizar novas estratégias de campanha, comunicação direta com seu eleitorado, mobilizar interessados e sondar opinião sobre questões pontuais.

O uso crescente do Twitter por políticos, jornalistas, estrategistas políticos e cidadãos o tornou parte importante de uma esfera em rede na qual questões políticas são negociadas publicamente. De acordo com Parmelee e Bichard (2012), a utilização do Twitter tem três objetivos que se destacam na vida política daqueles que seguem líderes ou atores políticos: 1) trata-se de uma forma rápida e sem filtros de obter informação política; 2) preenche o anseio dos usuários que desejam ser parte do processo político e não apenas recebedores de informação; e 3) é uma ferramenta de negócio para quem trabalha com política ou faz a cobertura de notícias políticas.

A maioria das publicações que relacionam o Twitter e a política é estrangeira, sobretudo norte-americana, mas já se observa um aumento das publicações brasileiras também² ${ }^{2}$

\footnotetext{
${ }^{2}$ Para aprofundamento, ver ROSSETTO et al., 2012.
} 
De acordo com uma ampla revisão bibliográfica que relata como os atores políticos utilizam o Twitter na comunicação política, é possível observar alguns tipos de uso mais comuns: como ferramenta de campanhas eleitorais (ARAÚJO; RIOS, 2010; BECHER; BRAGA, 2011; HONG; NADLER, 2012), mobilização e participação política (CERVI; MASSUCHIN, 2011; GOMES et al., 2009; SAYED, 2011), sondagens de opinião (CONNOR et al., 2010; GROSSE et al., 2012) e troca de informação política (BERRY, 2012; CREMONESE, 2010; HEMPHILL et al., 2013; TUMASJAN et al., 2010).

Quando utilizado como ferramenta de campanha eleitoral, uso mais relatado pela bibliografia, sites como o Twitter estão sendo aproveitados pelos políticos e governos para ampliar formas mais tradicionais de comunicação. Eles também usam as plataformas de redes sociais online para promover a comunicação com o público e entre agências (CHUN; WARNER, 2010). Alguns autores (BERTOT et al., 2010) vão ainda mais além e, de forma bem otimista, chegam a prever que os media sociais criarão uma cultura de abertura e transparência e, portanto, reduzirão a corrupção.

Na verdade aparentemente nenhuma outra tecnologia se tornou tão dinfundida no processo político com a rapidez que os media sociais tiveram entre 2010 e 2012. Num curto espaço de tempo, políticos das democracias modernas de todo o mundo adotaram avidamente essas ferramentas, vendo nelas poderosos meios para se engajar com seus constituintes (HONG; NADLER, 2012).

Quando observamos estudos brasileiros, a realidade se mostra semelhante. A maioria dos trabalhos na área são sobre situações de campanha (ROSSETTO et al., 2012). Um bom exemplo de relato desse tipo de uso é oferecido por Becher e Braga (2011) a respeito dos mecanismos de participação e deliberação online nas eleições brasileiras de 2010, apresentando resultados sobre o uso da internet pelos candidatos aos governos dos estados e ao Senado, a partir de uma metodologia adaptada para detectar padrões de uso da rede e mapear diferentes modalidades de interação entre candidatos e eleitores.

Partindo daquilo que a bibliografia já nos oferece, o trabalho aqui desenvolvido aplica o modelo teórico-metodológico do ETP à comunicação política no Twitter para investigar os usos políticos da plataforma. Nesta empreitada, vamos lidar com "fenômenos do universo político, tais como [...] campanhas eleitorais em sua vinculação com a decisão de voto e a 
opinião pública, que se estabelecem em relação direta com recursos, formas institucionais e meios da comunicação de massa" (GOMES, 2004, p. 44). O contexto de análise é a campanha eleitoral no Twitter, e a condição específica em investigação é como os indivíduos reagem aos tweets dos candidatos.

\section{Twitter, ETP e comunicação política}

Acredita-se que até aqui se tenha construído um terreno sólido para a ratificação da ligação entre o fenômeno do ETP e o campo da política, de forma geral. A ligação desses tópicos com o Twitter neste trabalho está na utilização da plataforma como lócus de aplicação empírica, após a verificação das características inerentes à plataforma, sua utilização para e pela política - relatada acima - e sua importância para o contexto comunicacional atual.

A razão da escolha do Twitter como objeto de estudo não está somente nos relatos da sua crescente utilização e importância para área, a plataforma se mostra potencialmente como um local de ressonância de temas e discussões políticas que são divulgadas pelos mais diversos meios de comunicação. Parece ser nos media sociais que as questões políticas repercutem e ganham diferentes desdobramentos; o que se acredita ter efeito na opinião dos indivíduos a respeito dos temas em discussão. Além disso, há ainda a caracterização desse tipo de ambiente como o que Chadwick (2009) e Wright (2012) chamam de third places ou third spaces, que são os espaços de discussão online onde o foco é essencialmente não político, mas onde emergem temas políticos dentro das conversações.

Um dos ganhos mais importantes da discussão da política nesses espaços é a possibilidade levar a política ao "local" onde as pessoas estão, e não tentar levar os cidadãos para espaços onde se discute somente questões políticas. Assim a exposição parece muito mais natural e semelhante àquela que ocorre no cotidiano presencial.

Além disso, a literatura nos mostra que um tipo de trabalho não encontrado na bibliografia nacional e ainda pouco explorado naquela estrangeira versa sobre os efeitos das mensagens políticas trocadas por meio das ferramentas sociais online. Por isso, também, a escolha por este caminho. 
O objetivo geral deste artigo é compreender os usos políticos do Twitter e como a opinião presumida pode influenciar a imagem que os indivíduos têm da comunicação política naquela plataforma, assim como as ações e reações dos eleitores ao conteúdo postado por candidatos. Interessa-nos especialmente o comportamento do cidadão enquanto audiência ativa desse meio de comunicação.

Atualmente, há um grande volume de pesquisas e análises sobre alguns dos temas vinculados aos objetivos elencados. A presente pesquisa foi elaborada tentando levar em conta esse quantitativo e todas as medidas utilizadas foram adaptadas de estudos anteriores (ANCU; COZMA, 2009; PARMELEE; BICHARD, 2012; ROJAS, 2010; SAYED, 2011). Portanto, para formulação das hipóteses e questões de pesquisa recorreu-se a essa literatura especializada, em particular àquela sobre a comunicação política nos SRS e sobre o ETP, o que nos levou a separá-las em dois blocos. Um bloco de hipóteses sobre os comportamentos e atividades no Twitter e outro sobre os efeitos presumidos nos usuários daquela plataforma.

\section{Hipóteses sobre comportamento e atividades no Twitter}

Sabemos que no Twitter cada perfil que um usuário acompanha é uma fonte de informação, no entanto, neste trabalho chamamos de fonte somente os candidatos que cada entrevistado segue. No contexto de estudo, temos diferentes tipos de candidatos nos mais variados quesitos, seja partido, gênero, histórico político, etc. Para ir direto ao que interessa e nos parece coerente com a pesquisa do ETP optamos por classificar as fontes em dois tipos: candidatos com quem o entrevistado tem alinhamento ideológico (chamados fontes afins ou candidatos semelhantes, que nos remete à noção de distância social do ETP) e candidatos de quem o entrevistado geralmente discorda politicamente (chamados de fontes divergentes ou candidatos discordantes).

Ao tratarmos de hipóteses sobre atividades no Twitter é preciso esclarecer o que queremos dizer com o termo. Essas atividades estão relacionadas ao uso dos recursos interativos da plataforma, que são mecanismos que utilizamos para nos referir a outro(s) usuário(s). 
Identificamos três recursos: 1) o retweet, que nos permite replicar uma mensagem recebida para aqueles que nos acompanham; 2) o reply, que é a ação de responder a um tweet; e 3) o mention, que nos permite mencionar um ou mais usuários em uma postagem. Acreditamos que a frequência com que cada pessoa utiliza esses recursos pode estar relacionada não só a forma como elas se envolvem com a política e com temas políticos (seu interesse ou histórico de participação, por exemplo), mas também com a sua relação com os candidatos que seguem. Destacamos essa relação - a ser entendida como quais os tipos de candidatos que o usuário acompanha, afins e/ou divergentes - sobretudo porque estamos tratando de um período eleitoral, no qual provavelmente há eleitores dedicados à campanha a favor de seu candidato ou contra seus opositores.

Isso nos leva a pensar a respeito das motivações que levam os cidadãos a utilizar o Twitter para a prática política. Neste trabalho, avaliamos três grandes razões de utilização desse SRS: para ser reconhecido, para influenciar os outros e para qualificar a própria opinião.

Reunindo as atividades no Twitter e os diferentes tipos de fonte, construímos duas suposições. Sabemos que nossos entrevistados podem seguir tanto candidatos (fontes) com quem eles têm afinidade de ideias políticas, quanto aqueles de quem eles discordam. $\mathrm{E}$ mesmo que eles não acompanhem ambos os tipos de fontes, esses usuários podem receber mensagens de uma fonte que eles não seguem, nem que seja inadvertidamente, por meio de qualquer perfil que eles acompanhem. Diante desse estímulo (uma mensagem de um candidato), há três possibilidades de uso de recursos interativos como reação: o retweet, a resposta e a menção (é claro que há também a possibilidade de não reação, mas essa não pode ser avaliada por meio da sondagem construída aqui). Sabendo disso, refletimos sobre como cada indivíduo vai reagir a depender de como ele se sente em relação à fonte da mensagem.

H1: As pessoas tendem a retuitar as mensagens das fontes com quem elas concordam politicamente e somente mencionar ou responder às fontes de quem elas discordam.

H2: O desejo de influenciar os outros está fortemente associado ao número de retweets da fonte com quem o entrevistado concorda. 


\section{Hipótese e questão de pesquisa sobre a presunção dos efeitos}

Uma variável muito importante para o teste do efeito é o tipo de conteúdo da mensagem, que por uma limitação metodológica não foi incluída neste teste. Em contrapartida, empregamos outras variáveis igualmente importantes e adequadas ao objetivo proposto. A falta da análise do conteúdo fez com que os conceitos de heterofilia e homofilia ${ }^{3}$ fossem a base para observar o efeito presumido a partir da distância social, ou seja, considerando os diferentes níveis de "outros". Assim, supondo que seja desejável ou conveniente ser influenciado por aqueles com quem concordamos e que seja indesejável ser influenciado por aqueles de quem discordamos, elaboramos nossa última hipótese.

H3: Os indivíduos vão achar que os outros são mais influenciados por fontes divergentes (candidatos discordantes) do que eles mesmos.

Encerramos esta seção com uma questão de pesquisa relativa ao efeito do efeito presumido. A QP1 combina esse efeito com conceitos já utilizados no bloco de hipóteses anterior sobre os tipos de fontes.

QP1: A influência estimada que os tweets dos candidatos têm sobre os outros e sobre o entrevistado varia de acordo com o tipo de fonte da mensagem?

\section{Resultados}

A pesquisa foi feita no formato de sondagem (survey) online com usuários do Twitter que seguiam candidatos a cargos eletivos, no contexto das eleições brasileiras de 2012. Os participantes foram recrutados através do Twitter com solicitação direta e técnica

\footnotetext{
${ }^{3}$ Para diferenciar a natureza do relacionamento entre emissor e receptor e tentar explicar os efeitos da interação entre candidato e eleitor, empregamos a concepção de homofilia e heterofilia (ROGERS; BHOWMIK, 1970). A homofilia se refere à similaridade de atributos entre dois indivíduos que interagem, é gostar do igual e foi utilizado para caracterizar os "outros" mais próximos do ego, chamados de laços fortes. A heterofilia se refere às diferenças, é gostar do diferente, e foi utilizado para identificar aqueles que o indivíduo considera socialmente mais distantes dele, os laços fracos.
} 
snowball ${ }^{4}$. O período para responder aos questionários foi de 15 dias, de 27 de novembro até 12 de dezembro de 2012, resultando em 142 respostas.

Do total de entrevistados $62,7 \%(n=89)$ são homens e 37,3\% (n=53) são mulheres, com faixa etária predominante dos 26 aos 35 anos (52,8\% dos entrevistados). No geral, o entrevistado médio é bem escolarizado, com ensino superior completo $(33,1 \%)$, ou pósgraduação incompleta $^{5}(28,9 \%)$, e renda familiar mensal de 4 a 10 salários mínimos $(42,3 \%)$ ou de 10 a 20 salários mínimos $(28,3 \%)$.

Os dados demonstram que esse público que segue políticos e candidatos no Twitter é bastante ativo nos SRS. O comportamento político online mais frequente foi a publicação de informação política $(85,9 \%)$, seguido pela publicação de informação de campanha $(69 \%)$.

Sobre as razões de uso da ferramenta, a maioria tem como objetivo influenciar os outros, seguido da razão de obter reconhecimento. $\mathrm{O}$ motivo menos importante de utilização desta rede social online é o de busca de informação e troca de razões para refinar a opinião ${ }^{6}$.

Para verificar qual tipo de fonte era mais seguida, as respostas foram limitadas à compreensão de quem seguia mais fontes afins, mais fontes divergentes ou o mesmo número de ambas. Neste caso observamos que $65,5 \%$ dos entrevistados relataram seguir mais fontes com quem têm alinhamento ideológico; 14,8\% seguem mais políticos e candidatos de quem geralmente discordam; e 16,9\% seguem aproximadamente a mesma quantidade de ambos.

Ainda sobre as fontes, foram verificadas as razões mais importantes que levam os entrevistados a seguir candidatos com quem eles geralmente compartilham ideias ou candidatos de quem geralmente discordam. No primeiro caso verificou-se que o motivo mais importante pelo qual eles seguem esses candidatos é o de, como se supunha, buscar

\footnotetext{
4 Técnica de amostragem que utiliza cadeias de referência, uma espécie de rede. É utilizada em pesquisas sociais nas quais os participantes iniciais de um estudo indicam novos participantes que por sua vez indicam novos participantes e assim sucessivamente, até que seja alcançado o objetivo proposto.

5 No questionário a pós-graduação foi identificada como mestrado/ doutorado.

${ }^{6}$ Esse resultado foi obtido com a matriz de correlação entre cada grupo de variáveis. Em todos os casos temos correlação superior a 0,3, que é o nível mínimo exigido para se continuar a análise fatorial, os testes de esfericidade de Bartlett com valor-p inferior a 0,05 e MSA maior que 0,5 .
} 
informação para apoiá-los $(73,3 \%)$. Em seguida vieram: para divulgar informação do candidato e, em terceiro lugar, para decidir qual candidato apoiar.

Sobre as fontes divergentes, é de se supor que indivíduos interessados em política se exponham a visões diferentes tanto para se informar quanto para contestá-las. Nesse sentido, os resultados corroboram com o previsto, a razão considerada pela maioria (44,3\%) como importante ou muito importante para seguir um candidato de quem se discorda politicamente é a de buscar informação para desafiar o candidato. Em seguida vêm: divulgar informação do candidato, participar de discussões com o candidato, decidir qual candidato apoiar, encontrar oponentes, e, por último, encontrar apoiadores.

\section{H1}

A primeira hipótese diz respeito ao tipo de fonte seguida pelo indivíduo e as suas reações diante de um tweet daquela fonte. No geral, os números são pouco expressivos e demonstram que os usuários reagem com pouca frequência aos tweets dos candidatos que seguem; tanto semelhantes quanto discordantes.

Os dados das fontes afins demonstram que, quando relacionado a esse tipo de fonte, o recurso interativo utilizado com mais frequência foi o retweet, relatado como ocorrido diariamente ou de 3 a 6 vezes por semana por $38 \%$ dos entrevistados. Esse resultado corrobora com o que se esperava. É claro que cada comportamento tem um intuito, que depende primeiramente do conteúdo da mensagem, entretanto, no geral, esse recurso conota endosso. Logo, para fontes com quem se compartilha pontos de vista semelhantes, se aguarda exatamente um reforço das ideias expressas. A tabela abaixo descreve melhor os resultados. 
Tabela 1: Frequência de uso dos recursos interativos do Twitter/ fontes afins

\begin{tabular}{|c|c|c|c|c|c|c|}
\hline & \multicolumn{2}{|c|}{$\begin{array}{l}\text { Respondeu } \\
\text { ao tweet de } \\
\text { um candidato }\end{array}$} & \multicolumn{2}{|c|}{$\begin{array}{l}\text { Retuitou um } \\
\text { candidato }\end{array}$} & \multicolumn{2}{|c|}{$\begin{array}{l}\text { Mencionou o } \\
\text { tweet de um } \\
\text { candidato }\end{array}$} \\
\hline & $\mathrm{N}$ & $\%$ & $\bar{N}$ & $\%$ & $\mathrm{~N}$ & $\%$ \\
\hline Diariamente & 12 & 8,5 & 21 & 14,8 & 11 & 7,7 \\
\hline $\begin{array}{l}\text { De } 3 \text { a } 6 \text { vezes por } \\
\text { semana }\end{array}$ & 20 & 14,1 & 33 & 23,2 & 33 & 23,2 \\
\hline Uma vez por semana & 28 & 19,7 & 27 & 19,0 & 24 & 16,9 \\
\hline $\begin{array}{l}\text { Menos de uma vez } \\
\text { por semana }\end{array}$ & 42 & 29,6 & 32 & 22,5 & 37 & 26,1 \\
\hline Nunca & 40 & 28,2 & 29 & 20,4 & 37 & 26,1 \\
\hline Total & 142 & 100 & 142 & 100 & 142 & 100 \\
\hline
\end{tabular}

Fonte: elaboração dos autores.

No caso das fontes divergentes, a frequência de reação é ainda menor, mas o tipo de reação relatado é coerente com o que se esperava deste tipo de fonte. Se observarmos os entrevistados que relataram alta frequência de suas reações (diariamente ou de 3 a 6 vezes por semana), temos 16,2\% mencionando candidatos; $14 \%$ respondendo-os; e 8,9\% retuitando-os. Apesar de poucos entrevistados utilizarem esses recursos com alta frequência, temos o retweet com a menor delas, o oposto do observado no caso anterior. Por outro lado, temos um alto percentual de entrevistados que disseram nunca ter utilizado nenhum desses recursos a respeito desse tipo de candidato. Isso significa que por mais que esses indivíduos sigam candidatos com quem eles geralmente discordam, eles pouco reagem aos seus tweets, e a reação menos reportada é exatamente a que conota endosso, não realizada nenhuma vez por $57,7 \%$ dos questionados. Portanto, os dados amostrais confirmam a H1. 
Tabela 2: Frequência de uso dos recursos interativos do Twitter/ fontes divergentes

\begin{tabular}{l|l|l|l|l|l|l|l}
\hline & \multicolumn{2}{|l|}{$\begin{array}{l}\text { Respondeu } \\
\text { ao tweet de } \\
\text { um candidato }\end{array}$} & \multicolumn{2}{l|}{$\begin{array}{l}\text { Retuitou um } \\
\text { candidato }\end{array}$} & \multicolumn{3}{l}{$\begin{array}{l}\text { Mencionou 0 } \\
\text { tweet de um } \\
\text { candidato }\end{array}$} \\
\cline { 2 - 8 } & $\mathrm{N}$ & $\%$ & $\mathrm{~N}$ & $\%$ & $\mathrm{~N}$ & $\%$ \\
\hline Diariamente & 9 & 6,3 & 8 & 5,6 & 7 & 4,9 \\
\hline $\begin{array}{l}\text { De 3 a 6 vezes por } \\
\text { semana }\end{array}$ & 11 & 7,7 & 9 & 6,3 & 16 & 11,3 \\
\hline $\begin{array}{l}\text { Uma vez por semana } \\
\text { Menos de uma vez } \\
\text { por semana }\end{array}$ & 19 & 13,4 & 10 & 7,0 & 12 & 8,5 \\
\hline Nunca & 35 & 24,6 & 33 & 23,2 & 44 & 31,0 \\
\hline Total & 142 & 100 & 142 & 100 & 142 & 100 \\
\hline
\end{tabular}

Fonte: elaboração dos autores.

\section{H2}

Para testar a H2 examinamos se há relação entre os diferentes motivos de utilização da plataforma e a frequência das reações reportadas às postagens de cada tipo de candidato. Como podemos observar na tabela 3, quase todas as relações são estatisticamente significativas, com valor-p<0.05. Só não encontramos associação entre: 1) a razão de utilização do Twitter para ser reconhecido com a frequência de retweets de fontes divergentes, o que significa que na busca por ser reconhecido não há sentido em retuitar fontes das quais discordo; e 2) entre a razão de influenciar os outros com a frequência de menções a candidatos discordantes, ou seja, mencionar fontes de informação das quais eu discordo não é uma ação que me possibilite influenciar minha audiência.

Observada a associação na maioria dos casos, atentamos para a sua força. Aqui ela é identificada pelo valor do qui-quadrado; quanto mais alto o valor, mais forte a associação. Os resultados nos testes nos permitem dizer que os indivíduos que utilizam o Twitter 
principalmente para influenciar os outros são, sobretudo, aqueles que seguem candidatos com quem eles concordam politicamente; e com tal objetivo eles, com mais frequência, retuítam as mensagens desses candidatos. Essa última afirmação nos é proporcionada pela análise das posições médias das frequências de retweets de postagens de fontes afins. Ela está detalhada na tabela 4 e significa que a média da razão de uso do Twitter para influenciar os outros é maior quando a frequência de retweets de fontes afins é diária. Assim confirmamos a $\mathrm{H} 2$.

Tabela 3: Razões de uso do Twitter $X$ frequência de uso dos recursos interativos da plataforma quanto às fontes (Kruskal Wallis)

\begin{tabular}{|c|c|c|c|c|c|c|}
\hline & \multicolumn{6}{|c|}{ AÇÕES DAS FONTES AFINS } \\
\hline & \multicolumn{2}{|c|}{$\begin{array}{l}\text { Frequência de } \\
\text { resposta tweet homo }\end{array}$} & \multicolumn{2}{|c|}{ Frequência RT homo } & \multicolumn{2}{|c|}{$\begin{array}{l}\text { Frequência de } \\
\text { menção homo }\end{array}$} \\
\hline & $\begin{array}{l}\text { Qui- } \\
\text { quadrado }\end{array}$ & $\begin{array}{l}\text { Asymp. } \\
\text { Sig. }\end{array}$ & $\begin{array}{l}\text { Qui- } \\
\text { quadrado }\end{array}$ & $\begin{array}{l}\text { Asymp. } \\
\text { Sig. }\end{array}$ & $\begin{array}{l}\text { Qui- } \\
\text { quadrado }\end{array}$ & $\begin{array}{l}\text { Asymp. } \\
\text { Sig. }\end{array}$ \\
\hline Para ser reconhecido & 23,982 &, $000^{*}$ & 20,008 &, $000^{*}$ & 10,429 &, $034^{*}$ \\
\hline Para influenciar... & 36,366 &, $000^{*}$ & 36,575 &, $000^{*}$ & 29,111 &, $000^{*}$ \\
\hline \multirow{4}{*}{$\begin{array}{l}\text { Para refinar minha } \\
\text { op... }\end{array}$} & 19,161 &, $001^{*}$ & 26,472 &, $000^{*}$ & 24,070 &, $000^{*}$ \\
\hline & \multicolumn{6}{|c|}{ AÇÕES DAS FONTES DIVERGENTES } \\
\hline & \multicolumn{2}{|c|}{$\begin{array}{l}\text { Frequência de } \\
\text { resposta tweet hetero }\end{array}$} & \multicolumn{2}{|c|}{ Frequência RT hetero } & \multicolumn{2}{|c|}{$\begin{array}{l}\text { Frequência de } \\
\text { menção hetero }\end{array}$} \\
\hline & $\begin{array}{l}\text { Qui- } \\
\text { quadrado }\end{array}$ & $\begin{array}{l}\text { Asymp. } \\
\text { Sig. }\end{array}$ & $\begin{array}{l}\text { Qui- } \\
\text { quadrado }\end{array}$ & $\begin{array}{l}\text { Asymp. } \\
\text { Sig. }\end{array}$ & $\begin{array}{l}\text { Qui- } \\
\text { quadrado }\end{array}$ & $\begin{array}{l}\text { Asymp. } \\
\text { Sig. }\end{array}$ \\
\hline Para ser reconhecido & 12,824 &, $012^{*}$ & 6,059 & ,195 & 10,949 &, $027^{*}$ \\
\hline Para influenciar... & 12,992 &, $011^{*}$ & 9,595 &, $048^{*}$ & 9,440 & ,051 \\
\hline $\begin{array}{l}\text { Para refinar minha } \\
\text { op... }\end{array}$ & 14,645 &, $005^{*}$ & 22,041 &, $000^{\star}$ & 19,070 &, $001^{*}$ \\
\hline
\end{tabular}

${ }^{\star}$ Associação estatisticamente significativa para $\alpha=0.05$. 
Fonte: elaboração dos autores.

Tabela 4: Rank da frequência de retweets de fontes afins

\begin{tabular}{l|l|l|l}
\hline & $\begin{array}{l}\text { Frequência de RT } \\
\text { homo }\end{array}$ & $\mathrm{N}$ & $\begin{array}{l}\text { Rank } \\
\text { médio }\end{array}$ \\
\hline $\begin{array}{l}\text { Para influenciar os } \\
\text { outros }\end{array}$ & Diariamente & 21 & 99,10 \\
\cline { 2 - 4 } & $\begin{array}{l}\text { De 3 a 6 vezes por } \\
\text { semana }\end{array}$ & 33 & 89,36 \\
\cline { 2 - 5 } & $\begin{array}{l}\text { Uma vez por } \\
\text { semana }\end{array}$ & 27 & 72,78 \\
\cline { 2 - 5 } & $\begin{array}{l}\text { Menos de uma vez } \\
\text { por semana }\end{array}$ & 32 & 58,84 \\
\cline { 2 - 5 } & Nunca & 29 & 43,97 \\
\cline { 2 - 5 } & Total & 142 & \\
\hline
\end{tabular}

Fonte: elaboração dos autores.

\section{Sobre a presunção dos efeitos - H3 e QP1}

$\mathrm{Na} \mathrm{H3}$ pressupõe-se que o efeito ocorra para fontes de quem o indivíduo geralmente discorda politicamente. Baseado na bibliografia do ETP supõe-se aí uma inconveniência (indesejabilidade) da mensagem que provenha de tal tipo candidato, logo se espera o efeito. A hipótese foi confirmada, ratificando não só o efeito como também que ele ocorreu em diferentes graus para diferentes níveis de "outros".

Para estabelecer a diferença da percepção de terceira pessoa, que é uma avaliação da influência desproporcional, foi gerado o gap de influência, subtraindo o quão influenciados 
os entrevistados perceberam os outros em geral (laços inexistentes) do quão influenciados eles perceberam a si mesmos (média=0.40, DP=1,21).

Como se pode ver na tabela abaixo, a média de influência relatada na primeira pessoa foi menor do que para quaisquer outros, mas, em desacordo com o que se verifica em estudos deste tipo, os laços fortes foram vistos como mais influenciados do que os laços fracos. Por outro lado, os laços inexistentes, ou seja, os outros que são completamente desconhecidos do entrevistado, foram percebidos como ainda mais influenciados; como se esperava.

Tabela 5: Influência percebida dos tweets/ fontes divergentes (estatística)

\begin{tabular}{l|l|l|l|l|l}
\hline \multicolumn{2}{l|}{} & $\begin{array}{l}\text { Entrevistado } \\
\text { influenciado } \\
\text { pelo candidato }\end{array}$ & $\begin{array}{l}\text { Laços fortes } \\
\text { influenciados } \\
\text { pelo candidato }\end{array}$ & $\begin{array}{l}\text { Laços fracos } \\
\text { influenciados } \\
\text { pelo candidato }\end{array}$ & $\begin{array}{l}\text { Laços } \\
\text { inexistentes } \\
\text { influenciados }\end{array}$ \\
\hline \multirow{2}{*}{$\mathrm{N}$} & Válido & 142 & 142 & 142 & 142 \\
\cline { 2 - 6 } & Missing & 0 & 0 & 0 & 0 \\
\hline Média &, 80 &, 99 &, 94 & 1,18 \\
\hline Desvio padrão &, 942 &, 942 &, 869 & 1,047 \\
\hline
\end{tabular}

Fonte: elaboração dos autores.

Ao observamos os números temos ainda outras interpretações, por exemplo: a maioria dos entrevistados alegando não ser influenciada pelos tweets desse tipo de fonte e seus laços inexistentes considerados muito influenciados. Relembramos que esses laços aqui só graduam a distância social entre os entrevistados e os diferentes "outros"; eles não são necessariamente aqueles que acompanham o perfil do entrevistado.

\section{QP1}

A QP1, que pretendida examinar se a influência estimada dos tweets dos candidatos sobre os indivíduos, varia de acordo com os diferentes tipos de candidatos (fontes). Neste caso 
estamos tratando de uma medida total de efeitos imaginados (ROJAS, 2010; SCHMIERBACH et al., 2008), criada com a soma das variáveis de influência percebida no entrevistado e nos outros em geral, para os dois tipos de fonte. A variável de influência percebida total para candidatos discordantes tem uma média de $2.04(\mathrm{DP}=1.80)$ e para candidatos semelhantes de $3.04(\mathrm{DP}=1.32)$. Portanto, em nível amostral, a influência percebida varia de acordo com o tipo de fonte, de forma que ela tende a ser maior para aquelas com quem o indivíduo geralmente concorda politicamente. Para aprofundamento, realizamos testes estatísticos para tornar possível a generalização do resultado, indo além da análise da nossa amostra. Com o teste de Wilcoxon, comparamos as médias dos índices totais de influência percebida e confirmamos que há diferença entre elas (valor-p<0.05), ou seja, a forma como a influência é percebida varia de acordo com o tipo de fonte.

\section{Conclusão}

Nossa nova realidade comunicacional, com crescente disponibilidade de informação (política) e a utilização das novas ferramentas digitais pelos atores políticos, gera novas necessidades e, com elas, desafios para a comunicação política em ambientes digitais.

É consenso que os políticos de, aparentemente, todas as democracias ocidentais estão cada vez mais adotando o Twitter, particularmente durante a época de eleição (GRAHAM et al., 2013). Parece que a ferramenta funciona principalmente como meio de alcançar potenciais eleitores e manter as relações sociais. Já como um canal para discutir questões políticas e trocar argumentos ele se mostra menos eficiente.

Se, regra geral, a teoria nos mostra que quando comparada à comunicação de massa a comunicação interpessoal é mais eficiente para influenciar decisões eleitorais (SCHIMITTBECK, 2004), temos nos media sociais o lugar ideal para esse tipo de estratégia. Neles temos uma associação de características inerentes à comunicação de massa - como informações que são produzidas por organizações de media e divulgadas em larga escala, por meio de um mecanismo unidirecional, para um público heterogêneo e anônimo - e predicados tipicamente da comunicação interpessoal - privada, que envolve um pequeno 
número de participantes, não mediada, bidirecional e que, geralmente, toma a forma de conversas pessoais. Por essas propriedades, a hipótese do ETP mostrou-se tão apropriada, já que ela se enquadra bem no conjunto de pressupostos teóricos que não mais entendem a comunicação mediada em oposição à comunicação interpessoal, mas aceita e assimila em suas suposições esses dois processos distintos como complementares e indissociáveis para o estudo dos efeitos.

Os resultados obtidos nos mostram que aqueles indivíduos que dizem ter um histórico de participação política maior, participam de forma mais ativa no Twitter e possuem um elevado nível de eficácia política interna, apesar de certa descrença nos políticos e no sistema político de forma geral. Aparentemente eles veem no Twitter uma ferramenta de disseminação de informação e exposição de opiniões, e o utilizam principalmente para tentar influenciar seus seguidores, postando informações e tentando oferecer à sua audiência pontos de vista diferentes sobre os assuntos políticos.

Esses usuários se consideram mais bem informados do que os outros em geral, e provavelmente até por isso se sintam na condição de informar terceiros que eles julgam menos informados. Essa é uma crença diretamente associada à percepção de terceira pessoa (third-person perception); e por mais que não tenhamos aqui testado o componente comportamental do efeito, podemos inferir que até essa necessidade de informar os outros seja resultado comportamental da percepção encontrada. Essa suposição se ratifica quando observamos que nossa amostra estimou que a maioria de seus seguidores é de totais desconhecidos.

Portanto, temos a seguinte situação: usuários do Twitter que acreditam que os outros são mais influenciados por tweets políticos do que eles mesmos, independente do tipo de fonte. Usuários esses que utilizam a ferramenta principalmente para tentar influenciar os outros, informando-os e demonstrando um ponto de vista diferente, e que têm em sua rede uma maioria de seguidores supostamente desconhecidos, isto é, mais socialmente distante, o que torna esses "outros" mais vulneráveis. Assim, uma maioria mais facilmente influenciável pelos outros e, de acordo com a bibliografia, que também pode ser influenciada por eles (usuários do Twitter), já que ela é predisposta e eles tuitam com esse objetivo. 
A respeito da relação dos usuários da ferramenta com os diferentes tipos de políticos, temos uma maioria que seguia candidatos com quem têm alinhamento ideológico. Nessa relação, destaque é dado também à influência percebida no indivíduo e nos diferentes tipos de outros. Verificamos a ocorrência da percepção de terceira pessoa para ambos os tipos de fontes, o que também ratifica a literatura da área.

Observamos, então, que a ação política no Twitter está fortemente ligada às percepções do indivíduo sobre como os outros serão afetados pelas diversas fontes de informação presentes na plataforma. Ao estimar um efeito forte no outro, e fraco em si mesmo especialmente de fontes divergentes -, o indivíduo tende a retuitar mensagens de fontes com quem têm alinhamento ideológico. Isso se dá numa ação de endosso àquela mensagem e numa tentativa de influenciar os outros da forma que lhe parece conveniente, ou seja, influenciá-lo a ter opiniões semelhantes às suas. Logo, as ações e os usos da plataforma estão estritamente relacionadas à percepção dos efeitos que as mensagens dos agentes políticos e candidatos têm no ego e nos outros.

Já que o fenômeno do ETP descreve a impressão de uma pessoa sobre os efeitos dos media, ele pode nos ajudar a explicar como a impressão a respeito da opinião pública no Twitter influencia, por exemplo, o processo de decisão eleitoral; como a opinião sobre a influência dos diferentes tipos de candidatos influencia o processo de disputa eleitoral; e como a utilização da plataforma pode influenciar o voto do indivíduo e seu comportamento político naquela rede - muito embora a dimensão propriamente eleitoral nesta pesquisa esteja limitada ao contexto de aplicação da sondagem. Isso significa que o domínio de como este efeito ocorre é informação valiosa para a elaboração de mensagens comunicativas que desejem persuadir a audiência; o que pode ser ainda mais valioso quando se trata de questões políticas.

É por essa razão, e por tantas outras evidenciadas também na literatura, que reconhecemos que as opiniões dos indivíduos a respeito dos outros desempenham um papel importante na formação e expressão pública de opiniões. Essa opinião das pessoas a respeito dos outros, de terceiros, é o efeito de terceira pessoa que aqui investigamos. É nesse ponto que reside nossa defesa a respeito do potencial que as impressões sobre os efeitos têm de moldar o comportamento político do indivíduo. 
A maior disponibilidade de informação política no ambiente digital gerou uma ruptura nos padrões convencionais de produção e consumo de conteúdo político e eleitoral. Algumas dessas rupturas podem ser claramente observadas no Twitter, entre elas o já citado fato de o usuário ter acesso a informações que, voluntariamente, não buscaria ou das quais não tomaria conhecimento, que chega até ele muitas vezes de forma inadvertida.

É claro que o uso do Twitter nas eleições abaixo do nível nacional permanece pouco pesquisado, sobretudo quando se trata da audiência. Em contrapartida, reconhecemos que as eleições que foram contexto deste estudo podem não ser o melhor cenário de análise para efeitos de uso e das mensagens que circulam no Twitter, já que a sua utilização tende a ser menor quando se vota em nível municipal do que no momento das eleições nacionais. Por outro lado, não acreditamos que esse tenha sido um entrave capaz de modificar os resultados obtidos. Primeiro, porque todos eles foram exaustivamente testados e validados estatisticamente. Segundo, e mais importante, porque quando se trata de efeitos, cognitivos ou comportamentais, o que importa de fato é o mecanismo que os gera, que ocorre nos indivíduos e sobre as suas representações no mundo mediado.

Nesse sentido, a relevância desta pesquisa está na compreensão e problematização dos desafios colocados à comunicação política em ambientes sociais digitais. Buscamos relatar e discutir algumas novas questões da comunicação política que emergem da inserção da política nesses ambientes, assim como se dá a organização e estruturação das interações sociais e políticas no Twitter e, finalmente, estudando como os processos de formação da opinião são influenciados por diversas variáveis nesses contextos.

Acreditamos assim ter contribuído para ampliação do conhecimento na área de comunicação política, ratificando a importância da plataforma para o cenário atual da comunicação, seus possíveis efeitos e seu papel na dieta informativa do cidadão e nas suas atividades relacionadas à discussão e prática política.

\section{Referências}

AGGIO, C. Dos websites aos media sociais: Apontamentos sobre os recursos e práticas de publicidade das campanhas eleitorais nos ambientes digitais. In: III Seminário Nacional de Ciência Política, 2010, Porto Alegre. Anais do III Seminário Nacional de Ciência Política, 2010. 
ANCU, M.; COZMA, R. MySpace Politics: Uses and Gratifications of Befriending Candidates. Journal of Broadcasting \& Electronic Media, 2009, v. 53, n. 4, p. 567-583.

ANDSAGER, J.L. et al. Perceived similarity of exemplar traits and behavior: Effects on message evaluation. Communication Research, v. 33, p. 3-18, 2006.

ANDSAGER, J. L.; WHITE, H. Allen. Self verus others: media, messages and the third-person effect. New Jersey: Lawrence Erlbaum Associates, 2007.

ARAUJO, G. F.; RIOS, R. Estratégias do Marketing Político Digital aplicadas à campanha presidencial de Barack Obama. In: XXXIII Congresso Nacional da Intercom, 2010, Caxias do Sul. Anais do XXXIII Congresso da Intercom, 2010.

BECHER, A.; BRAGA, S. Mecanismos de participação e deliberação on-line nas eleições de outubro de 2010 no Brasil. In: Seminário Nacional Sociologia \& Política, 2011, Curitiba. Anais do Seminário Nacional Sociologia \& Política, 2011.

BENNETT, W. L.; IYENGAR, S. A new era of minimal effects? The changing foundations of political communication. The Journal of Communication, v. 58, n. 4, p. 707-731, 2008.

BERRY, K. Presidential Campaign Politics, Public Opinion and Twitter: How Tweet It Is. 2012. Dissertação (Mestrado em Public Policy) - The Hubert H. Humphrey School of Public Affairs, The University of Minnesota, Minneapolis, 2012.

BERTOT, J. C. et al. Using ICTs to create a culture of transparency: E-government and social media as openness and anti-corruption tools for societies. Government Information Quarterly, v. 27, n. 3, p. 264-271, 2010.

CERVI, E. U.; MASSUCHIN, M. G. O uso do twitter nas eleições de 2010: o microblog nas campanhas dos principais candidatos ao governo do Paraná. Contemporânea, v.9, n.2, p. 319334, 2011.

CHADWICK, A.; HOWARD, P.N. Introduction: New directions in internet politics research. In: . Routledge Handbook of Internet Politics. Routledge: NY, 2009, p. 1-9.

CHUN, S.; WARNER, J. Finding information in an era of abundance: Towards a collaborative tagging environment in government. Information Polity, v. 15, n. 1-2, p.83-103, 2010.

COHEN, J. et al. Perceived Impact of defamation. An experiment on third-person effects. Public Opinion Quarterly, v. 52, n. 2, p. 161-173, 1988.

CONNOR, B. O. et al. From Tweets to Polls : Linking Text Sentiment to Public Opinion Time Series. In: IV International Conference on Weblogs and Social Media, 2010, Washington. Anais do IV International AAAI Conference on Weblogs and Social Media, 2010.

CREMONESE, D. Redes Sociais e Política no Brasil: a utilização do Twitter nas eleições 2010. IV Congresso Latino Americano de Opinião Pública da WAPOR, 2011, Belo Horizonte. Anais do IV Congresso Latino Americano de Opinião Pública da WAPOR, 2011.

CRIGLER, A.N. Introduction: Making Sense of Politics; Constructing Political Messages and Meanings. In: CRIGLER, Ann N. The Psychology of Political Communication. Michigan: The University of Michigan Press, 2001, p. 1-10. 
DAVISON, W. P. The Third-person effect in Communication. Public Opinion Quaterly, v. 47, p. 1$15,1983$.

EVELAND, W. P. et al. Rethinking the Social Distance Corollary: Perceived Likelihood of Expsoure and the Third-Person Perception. Communication Research, v. 26, n. 3, p. 275-302, 1999.

GOLAN, G. Moving beyond the perceptual component of the third-person effect: the influence of presumed influence on behavior. American Behavioral Scientist, v. 52, n. 2, p. 242-256, 2008.

GOMES, W. et al. Politics 2.0 : a campanha online de Barack Obama em 2008. Revista de Sociologia e Política, Curitiba, v. 17, n 34, p. 29-43, 2009.

GRAHAM, T. et al. Between broadcasting political messages and interacting with voters. Information, Communication \& Society, v. 16, n. 5, p. 692-716, 2013.

GROSSE, K. et al. Empowering an E-Government Platform Through Twitter-Based Arguments. Inteligencia Artificial, v. 50, p. 46-56, 2012.

GUNTHER, A.C. et al. The third-person effect. In: DONSBACH, W.; TRAUGOTT, M. (Eds.). Handbook of public opinion research. London: Sage Publications, 2008, p. 181-191.

HEMPHILL, L., et al. What's congress doing on twitter? In: 2013 Conference on Computer Supported Cooperative Work, 2013, San Antonio, TX. Anais do 2013 CSCW, 2013. Disponível em: <http://dl.acm.org/citation.cfm?doid=2441776.2441876>.

HONG, S.; NADLER, D. Which candidates do the public discuss online in an election campaign?: The use of social media by 2012 presidential candidates and its impact on candidate salience. Government Information Quarterly, v. 29, n. 4, p. 455-461, 2012.

LIM, M. Clicks, Cabs, and Coffee Houses: Social Media and Oppositional Movements in Egypt, 2004-2011. Journal of Communication, v. 62, p. 231-248, 2012.

McQUAIL, D. McQuail's Mass Communication Theory. London: Sage Publications, 2010.

MUTZ, D. The influence of perceptions of media influence: third-person effects and the public expression of opinions. International Journal of Public Opinion Research, v. 1, n. 1, p. 3-23, 1989.

Impersonal influence: How perceptions of mass collectives affect political attitudes.

Cambridge, England: Cambridge University Press, 1998.

PARMELEE, J. H.; BICHARD, S.L. Politics and the Twitter Revolution: How Tweets Influence the Relationship between Political Leaders and the Public. Maryland: Lexington Books, 2012.

PARVIAINEN, O. et al. Measuring the effect of social connections on political activity on Facebook. In: Conference of Internet, Politics and Policy, 2012, Oxford. Anais do IPP2012, 2012. Disponível em: $<$ http://microsites.oii.ox.ac.uk/ipp2012/papers $>$.

PAUL, B. et al. The Third-Person Effect: A Meta-Analysis of the Perceptual Hypothesis. Mass CommunicationCommunication, v. 3, n. 1, p. 57-85, 2000.

PERLOFF, Richard M. The third-person effect. In: BRYANT, J., ZILLMANN, D. Media effects: advances in theory and research. Lawrence Erlbaum Associates: London, 2002, p. 489-506. 
PERLOFF, Richard. Third-person effect research - 1983-1992: A review and synthesis. International Journal of Public Opinion Research, 1993, v. 5, n. 2, p. 167-184.

PRICE, V.; STROUD, N. J. Public attitudes toward polls: evidence from the 2000 U.S. Presidential election. International Journal of Public Opinion Research, v. 18, n. 4, p. 393-421, 2005.

ROGERS, E. M.; BHOWMIK, D. K. Homophily-Heterophily: relational concepts for communication research. Public Opinion Quarterly, v. 34, n. 4, p. 523-538, 1970.

ROJAS, H. "Corrective" Actions in the Public Sphere: How Perceptions of Media and Media Effects Shape Political Behaviors. International Journal of Public Opinion Research, v. 22, n. 3, p. 343-363, 2010.

ROSSETTO, G. P. N. et al. Twitter e comunicação política: limites e possibilidades. In: VIII Encontro da ABCP, 2012, Gramado. Anais do VIII Encontro da ABCP, 2012.

SALWEN, M.; DUPAGNE, M. The third-person effect: perceptions of the media's influence and immoral consequences. Communication Research, v. 26, n. 5, p. 523-549, 1999.

SAYED, N. Towards the egyptian revolution : activists ' perceptions of social media for mobilization. Journal of Arab \& Muslim Media Research, v. 4, n. 2+3, p. 273-298, 2011.

SCHARRER, Erica. Third-person perception and television violence: the role of out-group stereotyping in perceptions of susceptibility to effects. Communication Research, v. 29, n. 6, p. 681-704, 2002.

SCHMIERBACH, M. et al. Understanding Person Perceptions: Comparing Four Common Statistical Approaches to Third-Person Research. Mass Communication and Society, v. 11, n. 4, p. 492-513, 2008.

SCHIMITT-BECK, R. Political Communication Effects. The impact of mass media and personal conversations on voting. In: ESSER, F.; PFETSCH, B. Comparing political communication: theories, cases, and challenges. Cambridge: Cambridge University Press, 2004, p. 293-322.

TUMASJAN, A. et al. Predicting Elections with Twitter: What 140 Characters Reveal about Political Sentiment. ICWSM, v. 10, p. 178-185, 2010.

TUFEKCI, Z.; WILSON, C. Social Media and the Decision to Participate in Political Protest: Observations From Tahrir Square. Journal of Communication, v. 62, p. 363-379, 2012.

WILLIAMS, C.; GULATI, G. What is a Social Network Worth? Facebook and Vote Share in the 2008 Presidential Primaries. In: Annual Meeting of the American Political Science Association. Boston, 2008.

WRIGHT, S. Politics as usual? Revolution, normalization and a new agenda for online deliberation. New Media \& Society, 2012, v. 14, n. 2, p. 244-26. 


\section{O autor}

Graça Penha Nascimento Rossetto é jornalista, doutora em Comunicação e Cultura e Contemporânea (UFBA), professora titular do Centro Universitário Estácio da Bahia e assessora de imprensa do Complexo Hospitalar Universitário Professor Edgard Santos (UFBA). gracapnr@gmail.com 